\title{
2
}

\section{Induction and regulation of allergen-specific IgE}

\author{
Prescilla V. Jeurink ${ }^{\#}$ and Huub F.J. Savelkoul,
}

\begin{abstract}
The immune response is characterized by an initial rapid activation of the innate defence system, geared at recognizing common structures shared by many microorganisms. This innate immune response is a prerequisite to mount a highly antigenspecific adaptive immune response consisting of T-cell differentiation into effector subsets and B-cell differentiation into antibody-secreting plasma cells. Commonly, allergy is characterized by dendritic cells presenting allergenic peptides, activated Th2 cells producing signature cytokines like IL-4 and IL-5, and B-cells producing allergen-specific IgE antibodies. Under non-allergic conditions tolerance mechanisms, comprising regulatory $\mathrm{T}$-cell subsets, are suppressing potential immune responses to allergen exposure.

The protein structure of many allergens has been resolved and has provided an explanation for the epitope-specific IgE cross-reactivity responsible for the pollenfood syndrome. The knowledge on the protein characteristics of the major food allergens can provide better understanding why certain types of allergic symptoms can develop in particular individuals. Together with information on the genetic basis and the modulatory capacity of the environment, the Allergy Consortium Wageningen sets out to develop preventive measures to reduce allergic sensitization, to give advice to reduce allergen exposure and induce symptom reduction, and to provide clues to the management of an existing allergy.
\end{abstract}

Keywords: allergens; cross-reactive epitopes; Th2 cells; regulatory T cells; induction of IgE; food allergy

\section{The immune system}

The immune system is essential to protect us from infections with potentially harmful viruses, bacteria, fungi and parasites. The human body comprises on average about $2 \mathrm{~m}^{2}$ of skin, $80 \mathrm{~m}^{2}$ of lung and $350 \mathrm{~m}^{2}$ of gut tissue. This vast area of skin and mucosal tissue in the respiratory and digestive tract needs to be protected efficiently by the presence of a rapidly sensing and reacting immune system. The immune system has therefore evolved in a two-stage defence operation: the innate immunity or natural disease resistance and the adaptive or antigen-specific immune system. Both systems have their specific characteristics but work in concert to fight such infections. Here, we will discus parameters of the immune system that are relevant for the remaining chapters of this book. Further information on the immune system and the

\footnotetext{
\# Allergy Consortium Wageningen, Wageningen University and Research Centre, Wageningen, The Netherlands

*Corresponding author: E-mail: huub.savelkoul@wur.nl
} 
immunopathological basis of allergic diseases can be found in selected textbooks (Janeway et al. 2001; Leung et al. 2003).

\section{Innate immunity}

This system detects potentially harmful infections within minutes after entering the body, and its reaction is geared at rapidly diminishing the invader to very low numbers. This provides crucial time for the more effective but slower adaptive system to develop an antigen-specific adaptive immune response. Molecular patterns recognized by the innate immune system are shared by large groups of pathogens, and thus represent general patterns rather than specific structures. These invariant molecular patterns are conserved products of microbial metabolism, which are not subject to antigenic variability. The invariant molecular structures in microbial and foreign material represent main targets of innate immune recognition and are called pattern-associated molecular patterns (PAMP). Although the innate immune system selects against these PAMP, pathogens cannot modify or eliminate them because they are essential for the survival or pathogenicity of the micro-organisms. Any attempt to change PAMP will be lethal to the microbe or render it non-pathogenic. The overall effect of the innate immune recognition and the destruction of the target is that the recognized structures are of microbial origin and therefore distinct from 'self', i.e. the host. The major consequence of this requirement is the ability of the innate immune system to react selectively against micro-organisms. The features that are characteristic of PAMP can be demonstrated by several well-known examples of microbial modulators/stimulators of innate immune responses: (1) lipopolysaccharides (LPS) and teichoic acids are shared by, respectively, all gram-negative and grampositive bacteria; (2) unmethylated $\mathrm{CpG}$ motif is characteristic of bacterial DNA, but not as commonly by eukaryotic DNA; (3) mannans are conserved components of yeast cell walls.

\section{Humoral factors of innate immunity}

On host cells, PAMP may meet a number of pattern-recognition receptors (PRR). The main distinction of PRR from clonally distributed (and more specific) receptors of T- and B-lymphocytes is that their specificities are germline-encoded. Such receptors were present early in evolution and remained present by selection due to the presence of invasive micro-organisms at the population level. PRR are strategically expressed on cells which are the first to encounter pathogens during infection: epithelial cells and effector cells of the innate immune system (dendritic cells and macrophages). Currently, different serum proteins, including C-reactive protein (CRP), mannose-binding lectin (MBL) and LPS-binding protein, were shown to represent PRR. Moreover, PAMP upon binding to PRR induce in their target cells expression of several proinflammatory cytokines, like tumour necrosis factor (TNF)$\alpha$, IL-6, IL-12, IL-15, and type-1 interferons. Subsequently, inflammatory responses are modulated by the release of IL-10 and transforming growth factor (TGF)- $\beta$. In addition, pathogens will be bound to specific receptors expressed on leukocytes, especially when these pathogens are 'opsonized' by ligand molecules, like complement factors (C3), heat-shock proteins (hsp), LPS-binding proteins, etc. These ligand molecules were first identified in Drosophila flies and later also found in humans. These human receptors are called TOLL-like receptors (TLR) and these comprise now more than 10 family members. TLR belong to the class of pathogenrecognition receptors (PRR). These TLR are specific for structures present on bacteria, viruses and parasites and enable cells of the innate immune system which 
express these TLR to react rapidly to these infections. By recognizing the PAMP by the TLR this pathogen-TLR interaction plays a decisive role in the rapid noninflammatory clearance of infectious micro-organisms.

Antigens, like bacteria can be opsonized later on during the immune response with antigen-specific antibodies or complement factors. This opsonization facilitates their phagocytosis by macrophages and their subsequent intracellular killing and degradation. Binding of PAMP to relevant PRR will thus result in activation of the general innate NF- $\mathrm{KB}$ (nuclear factor in kappa light-chain-producing B-cells) transcription factor and subsequent production of pro-inflammatory cytokines, like IL-12. It is becoming increasingly clear that recognition of conserved microbial or viral antigens via 'non-specific' receptors, e.g. TLR, heat-shock proteins (HsP) or complement component $\mathrm{C} 3$ receptors present on cells of the innate immune system (natural killer (NK) cells, phagocytes), or recognition of microbial antigens by natural antibodies (NAbs) form the first and probably most important defence mechanism against infection. Besides activation of the innate arm of the immune system this release of IL-12 will also polarize the antigen-specifically activated Th-cell repertoire into preferential Th1 development (Figure 1). Such overactive and polarized Th1 repertoire potentially runs the risk of developing auto-immunity, but on the other hand evokes IL-12- and IFN- $\gamma$-dependent protection against certain infections.

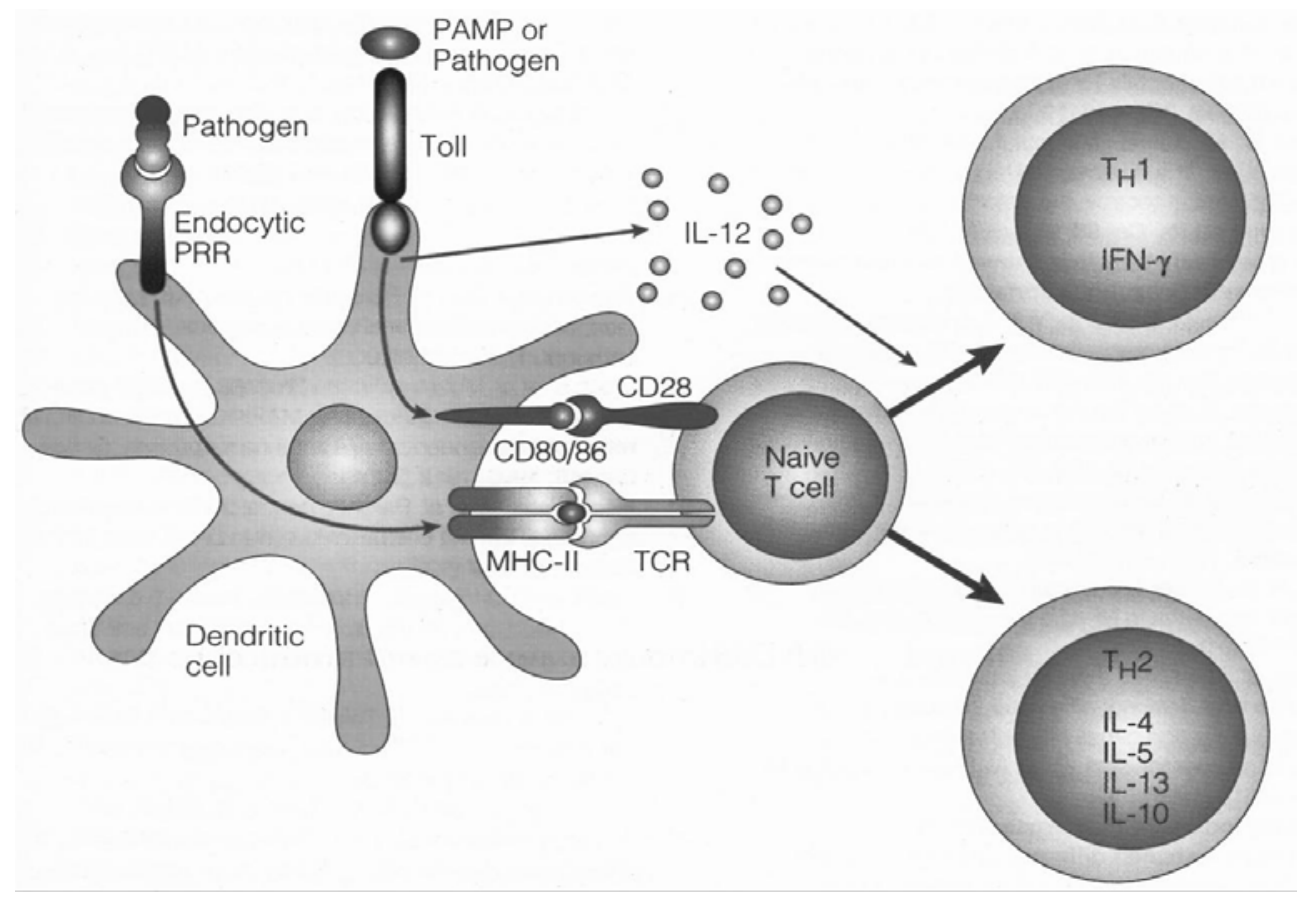

Figure 1. Schematic representation of the interactions between essential cell types in the antigen-specific adaptive immune response and the cross-talk with activities of the innate immune system. Interactions between infectious organisms carrying PAMP and PRR-bearing dendritic cells are shown and illustrate the interaction with T cells. (Modified after Medzhitov 2001)

\section{Adaptive immune response}

Naive CD4+ helper T cells (Th) develop into functionally mature effector cells upon stimulation with relevant antigenic peptides presented by major histocompatibility complex (MHC) class-II molecules on antigen-presenting cells (APC). Based on the characteristic set of cytokines produced, Th cells are commonly 
segregated into at least two different subpopulations: Th1 cells producing exclusively interleukin-2 (IL-2), interferon-gamma (IFN- $\gamma$ ) and lymphotoxin. Th2 cells, on the other hand, produce IL-4, IL-5, IL-6, IL-10 and IL-13. These Th1 and Th2 subsets appear to be extremes in cytokine production profiles, and within these polarized subsets, individual Th cells exhibit differential rather than coordinated cytokine gene expression. The Th- 1 and Th-2 subsets appear to cross-regulate each other's cytokine production profiles, mainly through IFN- $\gamma$ and IL-10. From this concept it was rationalized that disturbances in the balance between these two subsets may result in different clinical manifestations. IL-12 is a dominant factor promoting Th1 differentiation, and is produced by dendritic cells and macrophages. Moreover, IL-12 induces IFN- $\gamma$ production by $\mathrm{T}$ cells and natural killer (NK) cells. Recently, it was reported that IL-18 acts synergistically with IL-12 to induce Th1 development. Polarization of Th2 cells is critically dependent on the presence of IL-4 produced by Th cells, basophiles and mast cells. APC-derived IL-6 has also been shown to induce small amounts of IL-4 in developing Th cells. IL-10 and APC-derived prostaglandin E2 (PGE2) inhibit IL-12 production and Th1 priming. These Th1 and Th2 subsets are the extremes in a whole set of intermediately polarized Th subsets (Figure 2).

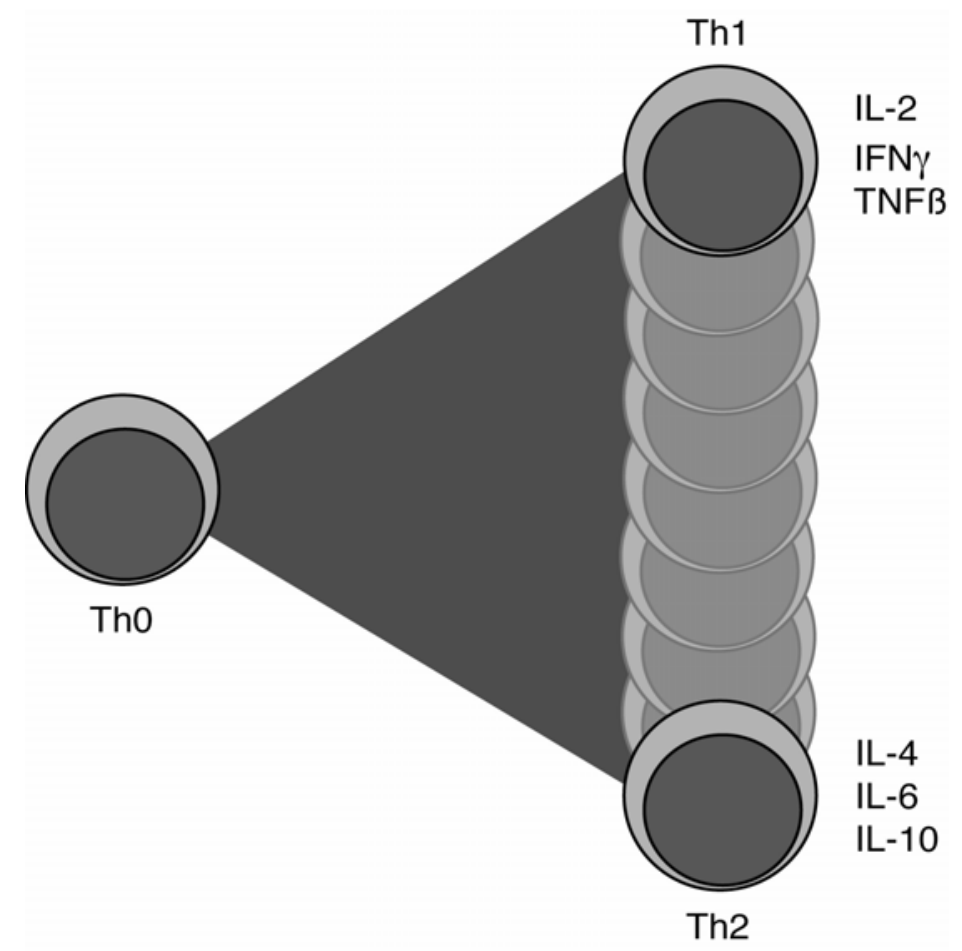

Figure 2. Effector Th1 and Th2 populations with their signature cytokines as differentiation end stages from activated Th0 cells. The figure shows that these Th1 and Th2 populations are the extremes in a whole cascade of intermediate subpopulations. All of these populations are characterized by the cytokine production profile they display after activation

Properties of many diseases, particularly systemic autoimmune disease, strongly support the involvement of helper T lymphocytes. For example, pathogenic autoantibody responses generally are of high-affinity IgG class, after having undergone affinity maturation, which requires helper $\mathrm{T}$ cells. The protein antigens to which many auto-antibodies are directed generally require T-cell help. Many of the successful therapies, e.g. cyclosporin A, act primary on $\mathrm{T}$ cells. Besides roles as helpers, $\mathrm{T}$ cells 
may directly provoke cellular injury during inflammatory phases of the disease process. $\mathrm{T}$ cells, and in particular $\mathrm{CD} 4^{+}$helper $\mathrm{T}$ cells, produce effector molecules, called cytokines, upon activation. A multiplicity of cytokine abnormalities has been associated with various autoimmune and immune-mediated diseases. It is thus becoming common practice to analyse the role of helper $T$ cells and the cytokines they produce in studying the immunpathological basis of particular diseases, to aid in the unambiguous diagnosis of the disease, and to provide parameters to monitor the efficacy of treatment.

Regulatory $\mathrm{T}$ cells, on the other hand, mediate active suppression of various immune responses (Figure 3). These T cells comprise classical Th2 cells, capable of inhibiting Th1 responses, but also alternative T-cell populations. The mechanism of peripheral tolerance has been focused mainly on the suppression of classical cellmediated Th1 responses and in animal (models of) diseases based on excessive activity of Th1 cells. It is now clear that such tolerance induction is also active in humoral type- 2 responses. One of the primary mechanisms of tolerance induction is via secretion of immunosuppressive cytokines, like IL-10, IL-4 and transforming growth factor $\beta$ (TGF- $\beta$ ). As mentioned before, regulatory T cells have been isolated from in-vitro cultures, which appeared to produce low levels of IL-2, no IL-4, but high levels of IL-10 and TGF- $\beta$. This demonstrates the importance of cytokines in regulating and dampening the immune response. It will thus be of crucial importance to determine whether the immunomodulating capacity of herbal and fungal polysaccharides in many diseases acts via induction of these regulatory T-cell subsets.

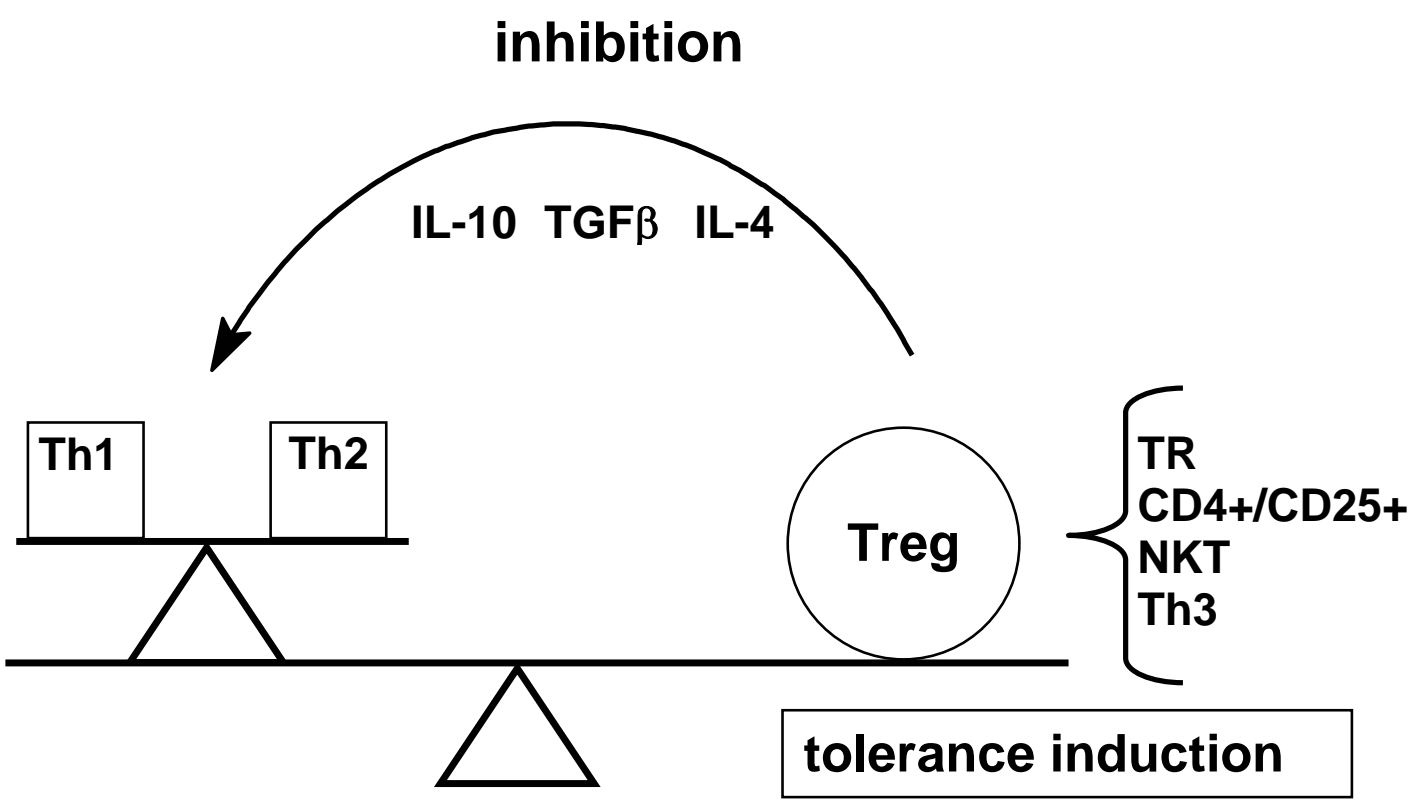

Figure 3. Contemporary view on T-cell regulation. Effector Th1 and Th2 populations are counterbalanced with regulatory T-cell populations (Treg). Several populations exist, including IL-10-producing inducible $\mathrm{T}_{\mathrm{R}}$ cells, natural occurring CD4+CD25+ populations, a rare population of IL-4-secreting Natural-Killer-like T cells (NKT) and TGF- $\beta$-producing Th3 cells that occur mainly in mucosal tissues. These soluble immunosuppressive cytokines can show their inhibitory activity on neighbouring cells and are considered to play an important role in tolerance induction in the T-cell compartment

$\mathrm{CD}^{+}$helper $\mathrm{T}$ cells play a crucial role in the regulation of the differentiation and activation of various T-cell subpopulations (Figure 4). These cells are able to conduct 
these functions by direct cell-cell contact through cell-surface interactions and by the release of soluble mediators, like cytokines.

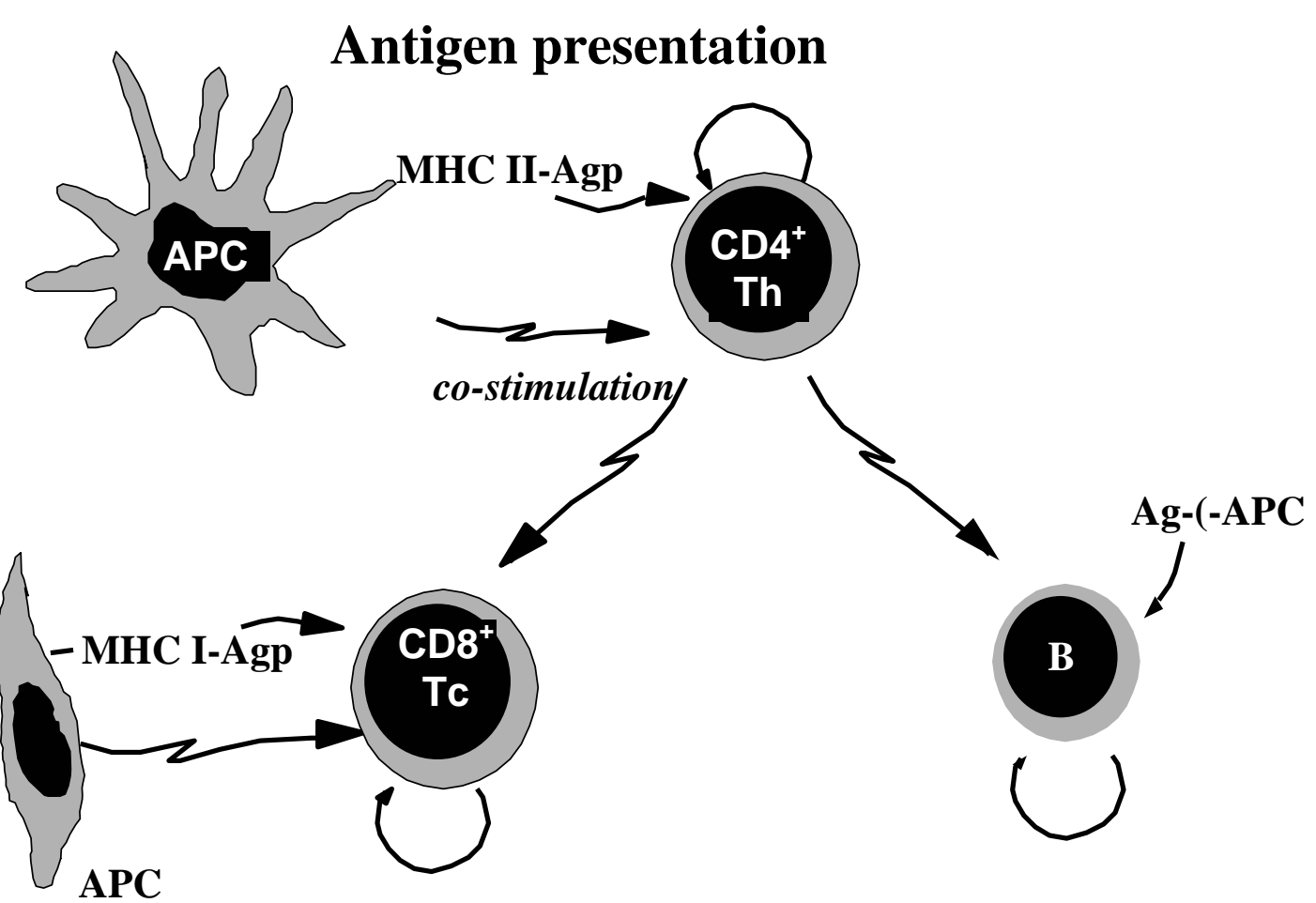

Figure 4. $\mathrm{CD}^{+}$helper $\mathrm{T}$ cells are in the centre of the regulation of effector functions of the immune response. These cells will help B cells to secrete antigen-specific antibodies and help cytotoxic $\mathrm{CD} 8^{+} \mathrm{T}$ cells to mature into killer cells, killing virus-infected cells and tumour cells. Th cells exert their helper function by surface interactions with other cell types and by the release of cytokines

In the vast majority of immune responses, the B-lymphocytes and T-lymphocytes that were activated in step 2 must be stimulated to proliferate by means of cytokines called interleukins (such as IL-2, IL-4, IL-5, Il-6 and IL-10), produced primarily by the activated CD4-helper lymphocytes. It is thought that in most immune responses, only around 1 in 1000 to 1 in 10,000 lymphocytes will have a receptor capable of binding the initiating antigen. After binding the epitopes, the lymphocytes must proliferate into large clones of identical cells (clonal selection). Thus, proliferation allows the production of clones of thousands of identical lymphocytes having specificity for the original antigen. This is essential to give enough cells to mount a successful immune response against that antigen.

The phenotypic responsiveness of animals, like humans, is determined by the genetic background modified by interactions with the environment. The genetic background is controlled by many different processes, ranging from Mendelian inheritance to epigenetically controlled expression of genetic loci. Breeding and selection for only a limited set of production parameters have potentially biased the genetically determined ability to mount a coordinated adaptive response to increasingly variable environmental factors. These factors include type of husbandry, food, infectious load, level of chronic stress, handling and transport, etc. Animals thus have to cope with profound changes in their environment that occur at multiple time points during their life: hatching or birth, weaning, fasting, transport, indoor vs. outdoor housing, etc. These rapidly changing conditions provide stress factors of 
multiple kinds precipitating increased disease susceptibility. Moreover, the robustness of these immune-compromised animals to withstand rapid and extreme changes in the environment is decreased.

The influence of nutrition on the health status of animals is increasingly considered to be important in providing humans with a safe and sustainable source of food. The concept of health status, particularly of farm animals, implies control of several different variables including welfare, (chronic) stress, infectious pressures, endocrine status, immune status, resulting in constant and high-end production parameters. The housing and feeding conditions of animals thereby provide important influences which exert their action by modulating the expression of the genetic background of the animal. As a result such animals display variable phenotypes having consequences for their 'health status' and production parameters. This variability is further increased due to the fact that at neonatal age the immune system is not fully developed yet. In particular, the important T-cell immune system (including the development of functional T-cell subsets and the cytokines they produce), which induces and regulates important processes during an immune response (humoral and cell-mediated protective immune response), is not yet fully active. During weaning, animals go through a period of fasting at their young age. This fasting period is considered to induce important and highly variable (genetically determined?) pro-inflammatory immune responses, based on cytokine production, which has consequences for the health status of these animals.

\section{Allergy}

The human immune system protects the body against harmful influences from outside (antigens). When an antigen enters our body, our immune system produces antibodies against those antigens. In an allergy the antigens are called allergens. An allergen itself is not harmful for the body; it is the reaction of the immune system that is harmful.

In allergy genetics play a critical role. Persons with an atopy have a genetic predisposition to get an allergic reaction after contact with an allergen. When there is a history of atopy in a family, a child has a higher chance to become allergic than without a history of atopy. With no atopic parents, the child has a $15 \%$ chance of becoming allergic, with one atopic parent it has a 35\% chance, and two atopic parents with the same allergy give a $75 \%$ chance of becoming allergic.

When an allergen intrudes the body, it is presented by an APC to naive Th0 cells. The APC produces IL-12 and $\mathrm{PGE}_{2}$ and directs the Th0 cells to become Th2 cells. The Th1-Th2 balance is disturbed, resulting in an overproduction of Th2 cells. Therefore, an allergy is called a Th2 disease. Th2 cells produce cytokines, especially IL-4, IL-5, IL-10 and IL-13. IL-4 and IL-10 inhibit the formation of Th1 cells. IL-10 also has a negative feedback on the production of Th2 cells. IL- 4 and IL-13 stimulate $\mathrm{B}$ cells to switch to IgE- and IgG4-antibody production. IL-5 activates and degranulates basophilic and eosinophilic granulocytes and mast cells. Secreted IgE attaches to high-affinity IgE receptors (FCERI) on mast cells, basophilic and eosinophilic granulocytes. During a second contact of the body with the allergen, it will be bound to the specific IgE on mast cells, basophils and eosinophils. After binding of the allergen and cross-linking of $\operatorname{IgE}$, mast cells, basophils and eosinophils will degranulate and inflammatory mediators (like histamine, leukotrienes and cytokines) will be released. Those inflammatory mediators cause allergic reactions like coughing, sneezing, redness and tearing (Figure 5). The role of IgG4 is still 
debated. The presence of IgG4 antibodies is often considered to be the result of chronic exposure to allergens. An allergy is characterized by the production of IL-4 and IL-13, which causes the development of specific IgE and IgG4 antibodies. Also the production of IL-5 is a characteristic of allergy, which causes an increased production of eosinophilic granulocytes and mast cells in the target organs of an atopic individual.

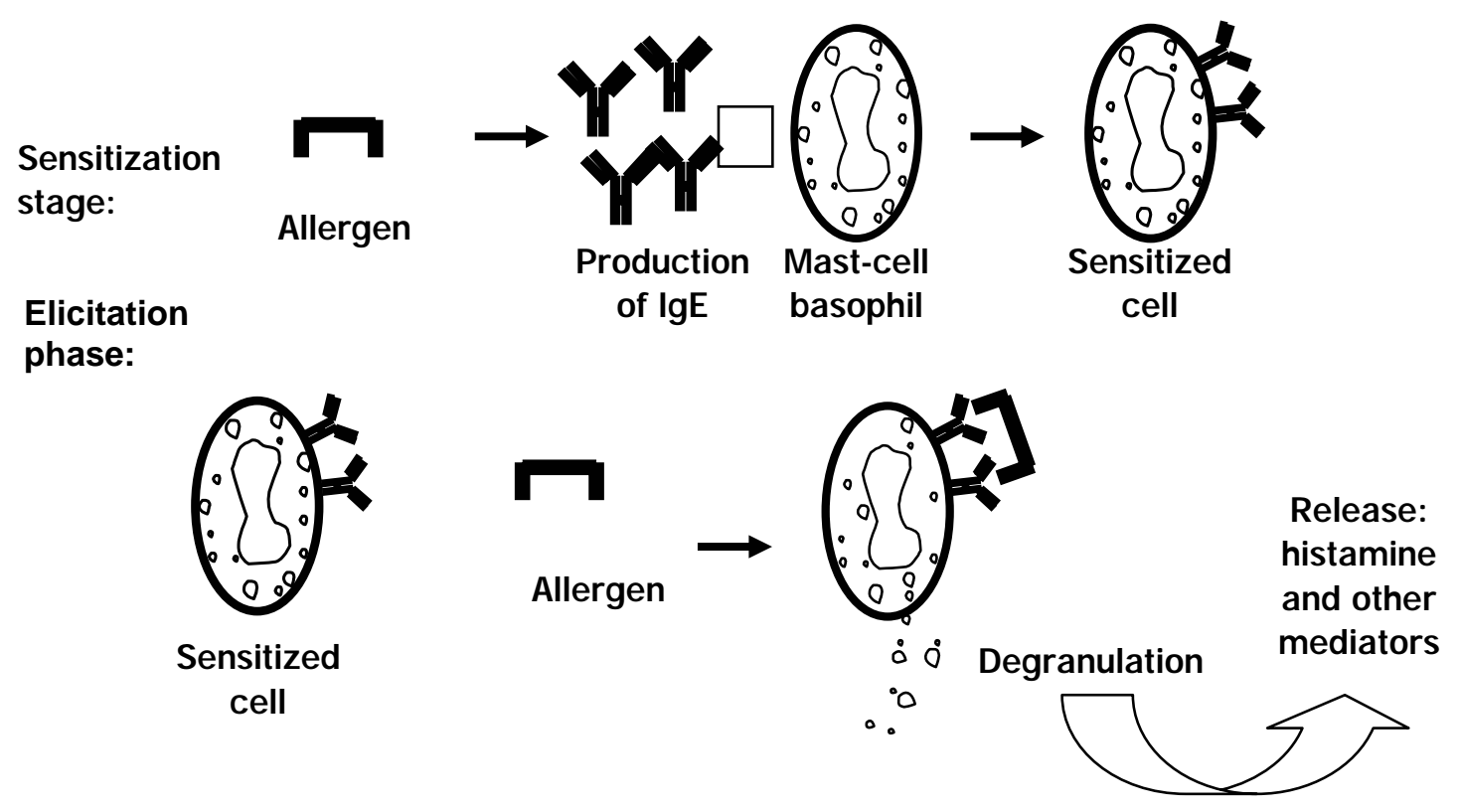

Figure 5. Schematic representation of the basic immune mechanisms of an immediate type-I allergic reaction, resulting in the rapid release of histamine and other substances inducing the typical clinical allergic pattern of symptoms

The figure above describes the elementary immunological principles underlying the induction of a food-allergic response. All the relevant cytokines, cell populations and effector functions are integrated. Allergic sensibilization describes the genetically determined propensity of certain individuals to react with the induction of Th2 cells and subsequent allergen-specific IgE-antibody formation upon repeated low-dose exposure of allergens at mucosal surfaces. This allergic sensitization can last for many months to years. Allergic sensibilization does not necessarily lead to immediate clinically overt allergic reactivity. Individuals sensitized against birch pollen can show no signs of birch-pollen-induced hay fever. Nevertheless, they have a high probability to develop food-allergic clinical symptoms due to the presence of cross-reactive $\operatorname{IgE}$ antibodies, recognizing homologous allergens in birch pollen (e.g. Bet v1) and certain foods, like apple (Mal d1), carrot (Dau c1), celery (Api g1), etc.

\section{Allergens}

Typically, allergens are protein antigens and allergens are widely present. Few proteins are allergens by themselves, but they can become allergens under special conditions or in susceptible persons. Allergens are individually relatively resistant to processing (temperature, pressure, vacuum, enzymes) and relatively digestionresistant. Currently, there is profound interest from the food industry to know the allergenicity of their products with respect to the new labelling requirements that are issued by EU regulations. 
An allergen molecule can carry one or more (up to roughly 25) epitopes, complexes of amino acids constituting potential binding site(s) for allergen-specific IgE antibodies. Many different allergens share similar sequence homologies that can explain cross-reactivity on the level of antibody binding. These sequences have specific binding motives in common, like the apricot sequence [Gly-X-His-Pro-X$\mathrm{Glu}$ ] in the IgE-binding epitope in peach [Gly-Ara-His-Pro-Gly-Glu]. Basically, we can separate linear epitopes consisting of 4-5 amino acids in a particular specific sequence. Adjacent amino acids constitute an IgE-binding epitope due to correct
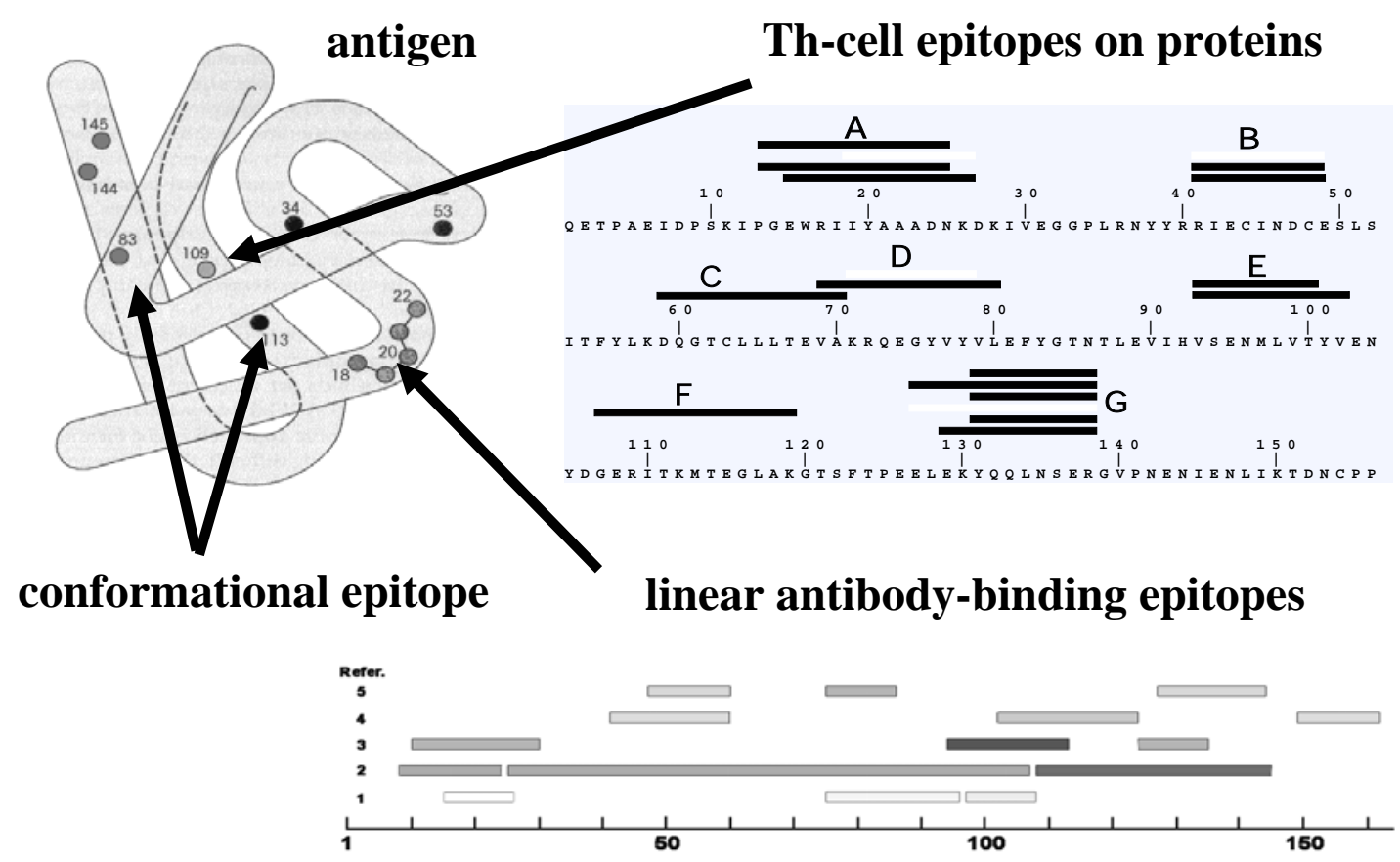

amino-acid number

Figure 6. Schematic representation of an allergen molecule carrying linear IgE-binding epitope (amino acids 18-22), a conformational epitope recognized by different IgE antibodies (amino acids 34, 53, 113 and amino acids 83, 144, 145). When comparing different antibody populations preferred epitopes shared by many antibodies become apparent. The antigen also carries T-cell epitopes that consist of preferred linear sequences of peptides that can be presented by antigen-presenting cells to specific $\mathrm{T}$ cells. A single amino acid responsible for the binding of a linear peptide derived from this allergen to a specific T-cell receptor is indicated (amino acid 109). When analysing different patients carrying IgE and $\mathrm{T}$ cells specific for this allergen, similar B- and T-cell epitopes are recognized and shared among these patients. From these sequences major B- and T-cell epitopes are known from various allergens

folding of the polypeptide chain. More than one binding site in multiple or linear sequences can be important for IgE binding, e.g., cod fish (Gad c1) consists of 4 linear epitopes (constituting DEDK and DELK) interspaced by 2 spacers of precisely 6 amino acids. Importantly the spacer length affects allergenicity of this allergen. Next, conformational epitopes exist that are conformation-dependent and therefore temperature- and processing-sensitive. Linear epitopes are enzyme-activity-sensitive, e.g. trypsin (Figure 6). We need to realize, however, that no specific structure or 
allergen can account for and predict the major allergenicity of a product, e.g. in milk, peanut, etc. Functional and/or structural homology is the reason for allergy! Protein abundance partially determines allergenicity, but not completely as is evidenced from the fact that the most abundant protein on earth, rubisco (photosynthetic ribulosebiphosphate carboxylase/oxygenase) is not an allergen!

In allergens also T-cell epitopes can be found that essentially consist of linear peptide sequences. These linear T-cell peptide sequences are generally different from peptide sequences comprising IgE-binding epitopes on the allergen. Only by comparison of amino-acid sequences of T- and B-cell epitopes of different patients will reveal the structure of these epitopes in allergens.

\section{IgE antibodies}

IgE antibodies appear in very low serum concentration $(0.00005 \mathrm{mg} / \mathrm{ml})$. These allergen-specific IgE antibodies can sensitize mast cells and basophilic granulocytes by binding via Fc portion to high-affinity receptor (FceR1). The ability of multivalent allergens to cross-link these bound IgE molecules will initiate the mediator release from these cells. Soluble IgE antibodies have a short half-life in serum (less than a day), but a markedly prolonged half-life (up to 14 days) when bound to Fc receptors. This binding thus protects IgE antibodies from proteolytic cleavage and clearance.

Like all antibody molecules also IgE antibodies are able to recognize two identical epitopes simultaneously (Figure 7). Unlike IgG molecules, however, IgE antibody molecules are rather inflexible. This poses restrictions to the three-dimensional orientation of the epitopes on the allergen molecule. This explains why the spacer length between the epitopes determines the allergenicity of the protein. For example: the allergen Gad c1 from cod fish carries 4 linear epitopes (bearing the amino-acid sequences DEDK and DELK), which are interspaced by 2 spacers of 6 amino acids. Different spacer lengths are directly proportional to the degree of $\operatorname{IgE}$ binding.

On the allergen molecule, the amino-acid residues responsible for the interaction with the specific IgE molecules can be shown in relation to the overall structure of the allergen. As shown from the front and the sides it is clear that the conformational but also linear IgE-binding epitopes can be present for IgE binding on a backbone of structural amino acids that ensure the proper three-dimensional structure of the allergen (Figure 8). 


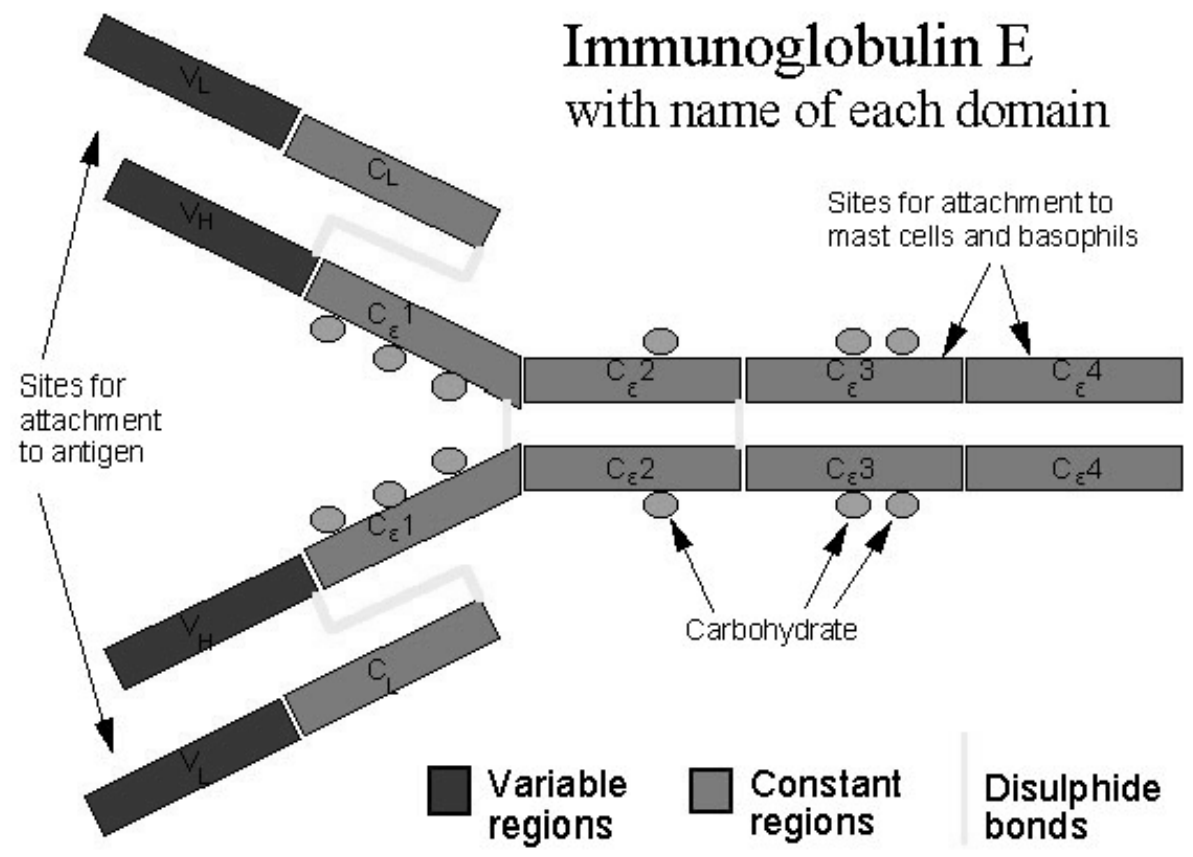

Figure 7. Schematic representation of the structure of an IgE molecule, showing the typical domain structure of the antibody molecule. The molecule consists of a single variable and multiple constant domains that carry carbohydrate residues. The two variable domains are involved in binding the two identical epitopes present on the allergen molecule. In the constant $\mathrm{Fc}$ part is involved in $\mathrm{Fc}$ receptor binding to mast cells and basophils
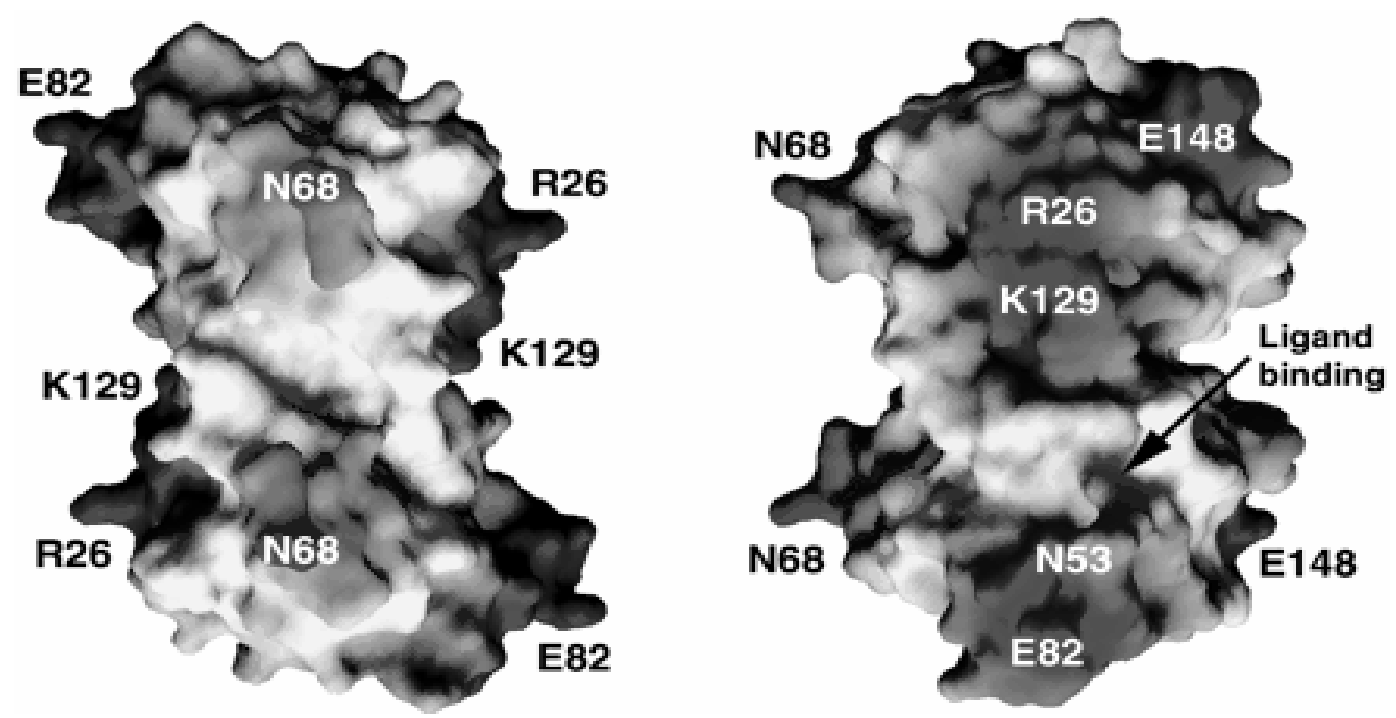

Figure 8. On the allergen molecule, the amino-acid residues responsible for the interaction with the specific IgE molecules can be shown in relation to the overall structure of the allergen. As shown from the front and the sides it is clear that the conformational but also linear IgE-binding epitopes can be present for IgE binding on a backbone of structural amino acids that ensure the proper three-dimensional structure of the allergen.

The amino-acid residues asparagine N68 are present on both subunits of the dimeric allergen, while the residues comprise the framework for proper binding. Especially the residues arginine R26, glutamic acid E82 and lysine K129 are responsible for the proper folding. In between, the ligand-binding site is shown and indicates that an allergen molecule might have particular properties, like acting as an enzyme binding the relevant substrate 


\section{Major food-allergen families}

In total about 129 food-allergen sequences are described in literature, distributed over 20 protein families of the 3849 possible families. More than $65 \%$ of the known food allergens belong to only four families: PR10, profilins, prolamines and cupins.

The pathogen-related proteins, like PR10, comprise a large family of homologous proteins with well-known members like Bet v1 from birch as well as Dau c1 from carrot, Pru av1 from cherry, Api g1 from celery and Mal d1 from apple. These proteins have functions in the defence of plants against infections with bacteria and fungi. From these allergenic proteins some 7 isoforms are described that can differ in only 1-5 amino acids. As explained above these minor sequence differences may have large implications on the IgE-binding ability. Despite their homology in amino-acid sequence (mainly the serine amino acid at position 122 is crucial) and therefore threedimensional structure (Figure 9), these proteins can behave similarly in a biochemical way. For example, Mal d1 and Api g1 are rather thermo-labile, while Dau c1, Pru av1 are relatively thermo-stable.

Profilins are abundant proteins of low molecular weight (14-16 kD) present in many plants and particularly in pollen of trees and grasses. In plants they perform functions pertaining to cytoskeleton activity (preventing actin polymerization), movement of cells and organelles, and a function in fertilization. Besides, these proteins are involved in intracellular signal transduction pathways by binding phospho-inositol-di-phosphate ( $\left.\mathrm{PIP}_{2}\right)$ and thus in activating signals for cells. In birch pollen, the Bet v2 protein is a profillin carrying the epitope [Cys-Ala-Leu-Ser-GlyGly-Tyr-Pro-Val-Cys] that occurs also in celery (Api g4). Sensitization via pollen (Bet v2), leads to cross-reactivity with fruit and vegetables in $15 \%$ of birch-pollensensitized patients. Bet v2-homologous sequences are present in soybean Gly m3 (78\%) and barley (75\%).
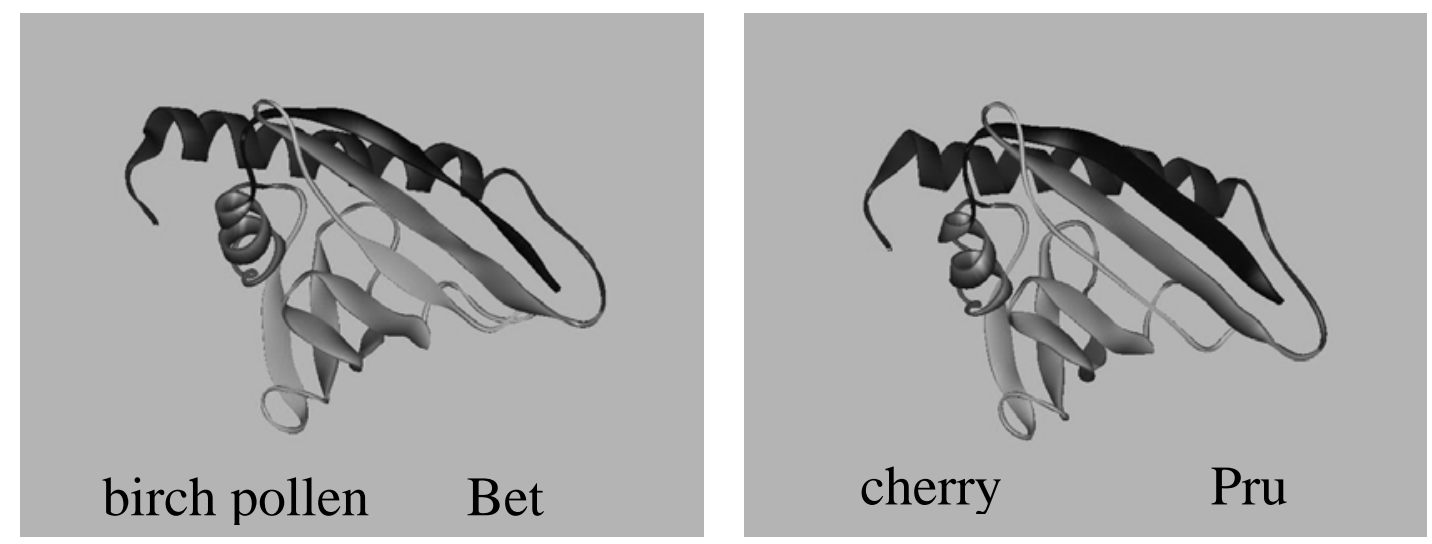

Figure 9. Structural homology between the major allergens in birch pollen and cherry: Bet v1 and Pru av1, respectively. Structural homology can explain cross-reactive IgE binding, but does not necessarily imply similar chemical stability or activity in the induction of allergic symptoms in allergic individuals

The prolamin superfamily consists of subgroups, called $2 \mathrm{~S}$ albumin, like Ber e1 (Brazil nut); Ses i2 (sesame); cereal prolamines, like Tri a19 (wheat); Sec c20 (rey); cereal $\alpha$-amylase/dimeric amylase inhibitor playing a role in Baker's asthma. These small proteins $(7-16 \mathrm{kD})$ are alcohol-soluble storage proteins in cereals that have a similar 3D structure. The cupin superfamily consists of vicillins (like Ara h1 in peanut and Jug r2 in walnut) and legumins (Ara h3 and Ara h4 in peanut and Cor a9 in hazelnut). These are multimeric (3-6 subunits) complexes of monomers $(50-60 \mathrm{kD})$ 
that are folded into a characteristic barrel structure. These proteins share short conserved-sequence motifs (RDPYSP) and are unusually stable to denaturation and proteolysis. Lipid-transfer proteins (LTP) are anti-fungal defence proteins in plants; they are heat-stable and acid-resistant. An example is Mal d3, which has 4 intra-chain cysteine bridges. They are present in all plants and many animals and constitute major allergens in apricot, plum, peach and cherry (Pru av3). Cross-reactivity has been described between LTP of peach and maize, which are highly unrelated sources! Cross-reactive carbohydrate determinants (CCD) can be found in a wide variety of allergenic materials, including pollen, foods, insect venoms, shellfish, etc. Generally these CCD display high IgE binding, but generally very low clinical relevance.

As stated above, the level of cross-reactivity in $\operatorname{IgE}$ binding does not necessarily correlate with the clinical reactivity. This holds actually true for many individuals in which IgE antibodies are present that bind particular proteins and, in the case of allergy, correlate with the clinical disease pattern. Such antibodies can cross-react with other allergens based on overall structural homology, primary amino-acid sequence homology, and presence of sequence motifs constituting IgE-binding epitopes. Such cross-reactivity does not always reflect clinical allergenic reactivity. This necessitates additional laboratory analysis of the fine specificity of IgE antibodies, generally by inhibition-type binding assays.

\section{Clinical aspects}

Immunogenetic basis

As stated above, allergic diseases are considered to be Th2-driven immunopathologies. Thus, several Th2-linked genes were analysed for the presence of promoter polymorphisms or exon mutations occurring in patients. Such polymorphisms or mutations could be responsible for the risk to develop allergic symptoms and provide possible markers predicting allergic diseases. Moreover, they can be used to monitor the development of allergic symptoms and potentially effective treatment. So far, several exon mutations in cytokine genes were found in allergic patients: IL-4Receptor (Q576R, I50V), IL-10 (C-571A), TNF- $\alpha$ (C368A), CD14 (C159T), FceRIB (I181L, I183V, E237G). Promotor polymorphisms were described for TNF- $\alpha$ (G-308A), IL-4 (C-590T), IL-10 (-3575T/A, -2849G/A, 2763C/A, -1082A/G, -819T/C and -592A/C), and IL-9 (A-345T).

Many of these genes are located in defined positions on several different chromosomes and genetic linkage has been proposed in allergic individuals with several chromosomes, like 5q3-33 (cytokine gene cluster), 5q32 (beta-2 receptor), 6p21.3 (TNF- $\alpha$ ), 11q13 (FcERIB), 12q15-24.1 (IFN- $\gamma$ ) and 1q32.1 (IL-10). Despite these interesting linkages, the multigenic nature of allergic diseases and the variability in disease expression among affected individuals, these data are not yet validated in large populations or used on a routine basis.

Analysis of family history for allergic diseases can help in identifying young children at risk of developing allergy. The presence of allergy in the mother or the presence of one allergic sibling increases the risk for the newborn from $15 \%$ to $35 \%$, while allergy in the father increases the risk to $25 \%$. The presence of allergy in both parents and if both parents suffer from the same allergic-disease expression increases the risk to develop allergic symptoms in a newborn to $75 \%$. 


\section{Environmental basis}

The genetic propensity to put an individual at risk of developing allergic disease is modulated by exposure to environmental factors. Such factors include pollution of air and water, infectious load and vaccination strategies, globalization of foods and exposure to novel foods, induced chronic stress levels, level of exercise and many others. Some of these factors are known for their added risk, some can be influenced by choosing an active, less allergenic life style. Some factors, however, cannot be influenced by individual action, and these are hard to control. The choice of life style and the ability to control this with respect to modulate the risk to develop allergic diseases requires individualized advice and proper information. This is where preventive measures can help.

\section{Clinical symptoms in patients at allergen exposition}

The allergic march describes the age-related difference in expression of allergic symptoms from newborns to adults. It is widely believed that most babies display their first symptoms of allergy by suffering from food allergy within the first few months of their life. Generally this allergy is reflected as eczema. After 1-2 years of age, in most children this eczema resolves (60-70\%), giving rise to respiratory symptoms in some $(30 \%)$, while the rest $(30 \%)$ remains symptom-free for years or even for life. At 6 years of age allergic asthma is prevalent in children at risk to develop allergy, while at 12-16 years of age it is primarily hay fever. Although still widely accepted there are data to support that heterogeneity might exist in allergic children with some displaying skin-related symptoms (eczema) while others display respiratory-system-related symptoms (asthma and hay fever).

Allergic disease can be expressed in the skin (atopic dermatitis: 10-12\% of patients), gut (food allergy: 20\%), the nose and eyes (rhinoconjunctivitis, hay fever: 10-20\%), and lungs (allergic asthma: 2.5-10\%). Allergic diseases require an effective diagnosis that can predict the identification of individuals at risk for allergic sensibilization. The genetic risk to develop allergic disease can be determined by analysing family history and the presence of polymorphisms or mutations in selective cytokine genes. The individual behaviour of an individual at risk and the quality of life that allergic persons want to achieve determine to a large extent the extent and severity of allergic diseases. Unfortunately, the perception of allergic disease by the presence of clinical symptoms and diagnostic data might be different for various allergic diseases, especially for food allergy. Also the difference between IgE- and non-IgE-mediated allergic diseases might compromise proper advice, treatment and management of the allergy.

\section{Prevention}

Prevention of allergic symptoms is of crucial importance in individuals at risk. This can be achieved at multiple levels. Primary prevention should provide the prediction of being at risk and is based on general recommendations for society, as well as on individual recommendations for individual people. These recommendations comprise breast-feeding to children from 4 to 6 months, and, if this is not possible, to provide newborns at risk with hypoallergenic-formula milk. Also late addition of solid food (after the 4th month), avoidance of pollutants (traffic exhaust), avoidance of indoor allergens (e.g. pets) and avoidance of irritating/sensitizing skin contacts together should decrease the pace of development and the severity of symptoms in allergic disease. Secondary prevention should be based on screening for IgE-mediated sensitization by blood assays and skin tests. This analysis should provide 
recommendations for sensitized individuals or patients with one allergic disease. Advice comprises allergen avoidance (in home, restaurant and school), pharmacotherapy (e.g. antihistamines, Epipen) and immunotherapy (successful for inhalant allergens, but not for food). Tertiary prevention comprises post-treatment follow-up and patient management, and is based on adequate post-treatment follow-up (including skin care, psychosomatic counselling), educational programmes (asthma/eczema schools), label reading (especially urgent with new EC directives), the development of a concept of patient management, and rehabilitation in special clinics/programs.

\section{Conclusion}

Allergic diseases have an immunological component which is not only involved in sensibilization but also in the interaction between the genetic background and the modulation by the environment. Ultimately, a chronic inflammatory condition is apparent in an individually determined target organ linked to exposure with allergens, like skin, lungs, upper respiratory system and the gastro-intestinal tract. Allergic symptoms will subsequently develop, including oral allergy syndrome, eczema, hay fever and allergic asthma. These symptoms will develop after exposure to allergens, pollen, house dust and food proteins. Environmental conditions will modulate the disease's expression; they consist of factors like tobacco smoke, air pollution, infections, vaccinations, food, exercise and factors linked to the profession. Knowledge of these principles, obtained in a multidisciplinary setting can provide advice and information on prevention of allergic diseases, both for the individual at risk and the society.

\section{References}

Janeway, C.A., Travers, P., Walport, M., et al., 2001. Immunobiology: the immune system in health and disease. 5th edn. Garland Science, New York.

Leung, D.Y.M., Sampson, H.A., Geha, R.S., et al., 2003. Pediatric allergy: principles and practice. Mosby, St. Louis.

Medzhitov, R., 2001. Toll-like receptors and innate immunity. Nature Reviews. Immunology, 1 (2), 135-145. 